\title{
Comparison between urethrostomy and penile resection for treatment of congenital penile urethral dilatation in calves
}

\author{
A. M. Sedeek ${ }^{1}$, H. A. Bakr ${ }^{2 *}$ \\ ${ }^{l}$ Department of Surgery, Anesthesiology and Radiology and ${ }^{2}$ Department of Animal Medicine \\ Faculty of Veterinary Medicine, Beni-Suef University, Beni-Suef 62511, Egypt.
}

\begin{abstract}
A clinical study was conducted under field condition during the period from January 2005 to December 2008 in Beni-Suef province on a total number of 15 calves suffered from congenital penile urethral dilatation at the perineal region. The affection was diagnosed and treated surgically under field condition and treatment included perineal urethrostomy (8 calves) and partial resection of penis including the dilated urethra ( 7 calves). Results revealed that duration of surgery for urethrostomy was shorter (mean 36.25 minutes) than penile resection (mean 85.71 minutes), bleeding was so extensive in penile resection group than urethrostomy one and cost of surgery was lower for urethrostomy than penile resection but aftercare cost was twice that of penile resection. Complications were higher in urethrostomy group, recovery period was nearly the same in both groups (14.37 and 14.28 days), mean body gain was higher in penile resection group ( $82 \mathrm{~kg}$ ) than urethrostomy group $(77.14 \mathrm{~kg})$, and both groups considered of low quality animals at market as a result of loss of breeding capability and presence of urine scald. It could be concluded that both techniques are satisfactory but partial penile resection including the dilated urethra is superior to urethrostomy despite the higher cost and invasive surgical procedure.
\end{abstract}

Congenital urethral dilatation in calves is an uncommon affection that infrequently discussed in scientific veterinary literatures and texts. The condition occurs sporadically and is recognized easily, but sometimes it is neither so easily repaired, nor the outcome of its surgical correction has been fully discussed (Johnson, 1980; Karras et al., 1992; Geccelep and Alkan, 2000; King et al., 2002; Misk, 2008).

Dilatation is defined as the extension of cavitary organs in every direction and it is either congenital or acquired. Urethral dilatation is observed at the proximal perineum spot in heifers and adults, and emerges as a result of the transient urethral obstruction, or much less frequently due to bacterial urethritis of the lower urinary section (Tharp and Venzke, 1954; Karras et al., 1992; Anderson et al., 1993; Gasthuys et al., 1993).

The condition can be observed few days after birth or even up to many months later (Anderson et al., 1993; GeCcelep and Alkan, 2000; King et al., 2002). Clinical signs included presence of large bladder-like mass protruding from the perineal midline with difficult

\footnotetext{
* Corresponding author. Tel.: +20 0822322066; fax: +20822327982 .

E-mail address: hossam.bakr@bsu.edu.eg (Hossam Bakr).
}

urination. Differential diagnoses include cutaneous cyst, ectopic urinary bladder, urethral diverticulum (dilatation), and rupture of the urethra at the level of ischial arch (Anderson et al., 1993; King et al., 2002).

The condition is usually associated with urethritis and/or cystitis as a result of accumulation of urine in the swelling for a long period (Anderson et al., 1993; Parsons et al., 1998), and even rupture of the urinary bladder may ensue if the condition was associated with aplasia of the penis (Javdani et al., 2009).

Treatment of such cases was directed towards surgical correction when it is possible (Karras et al., 1992), otherwise perineal urethrostomy or penile resection is indicated if infection is refractory to conservative management. However, success rate of surgical treatment by urethrostomy varied from $37.5 \%$ in eight cases to $100 \%$ in one case (Gasthuys et al., 1996; GeCcelep and Alkan, 2000). Moreover the over all success rate of urethrostomy, either short term or long term, was not satisfactory in many literatures (Haven et al., 1993; Van Metre et al., 1996) as it may be followed with stricture of the fistula orifice or cystitis (Stone et al., 1997).

The aim of the present work is to evaluate two surgical techniques (the traditional perineal urethrostomy and partial penile resection including the dilated urethra) for treatment of 
congenital penile urethral dilatation bin calves and their long-term survival, cost and outcome.

\section{Materials and Methods}

The study was conducted during the period from January 2005 to December 2008 in BeniSuef province on a total number of 15 calves suffered from congenital penile urethral dilatation at the perineal region.

Animals. The subjects of the study were 15 calves, 6-12 months and 60-120 kg live body weight and these animals were treated under field condition. Clinical examination of these animals was performed according to Kelley (1984).

History and clinical signs. These animals were purchased from markets with perineal swelling and had episodes of fluctuating fever that partially respond to antimicrobial treatment. Affected animals had circumscribed fluctuating swelling that appeared in mid-perineal region ventral to the anus and extended over the perineal region till the scrotum. The size of the swelling varied from $10-20 \mathrm{~cm}$ in diameter with no pain reaction at palpation and squeezing revealed exudation of whitish yellow fluid from the prepuce orifice. Stimulation of urination caused enlargement and tendering of the swelling that was followed by continuous passive drippling of turbid whitish yellow urine. Aseptic exploratory puncture of the swelling revealed presence of fluid of the same mentioned physical characters and ammoniacal odor.

Surgical treatment. Affected animals were classified into two groups, group (I) consisted of 8 animals was subjected to urethrostomy (Figs. 1-5) and group (II) consisted of 7 animals was subjected to partial penile resection including the dilated urethra with establishment of urethral fistula below the anus (Figs. 6-10).

Group I. These 8 calves were subjected to urethrostomy in the following manner. Animals were subjected to caudal epidural analgesia by using lidocaine $\mathrm{HCl} 2 \%$ and sedation by intramuscular xylazine $\mathrm{HCl} 2 \%$ in a dose rate of $0.22 \mathrm{mg} / \mathrm{kg}$ (Hall and Clarke, 1981). The animals were positioned and restrained in lateral recumbency and the seat of surgery was prepared for aseptic surgery in routine manner, then after an elliptical incision 5-7 cm length and $3 \mathrm{~cm}$ width was created at the lowest point of the swelling just dorsal to the scrotum (Fig.1-5). The incision was advanced to open the swelling after which the contents of the swelling were evacuated and the cavity was flushed with mild antiseptic solution (povidone iodine 10\% diluted
$50 \%$ with saline). The wall of the urethra was sutured to the skin with simple interrupted silk stitches in order to create urethral fistula.

Aftercare included daily flushing of the swelling with the mild antiseptic, dressing of the wound, and systemic long acting tetracycline $20 \% 1 \mathrm{ml} / 10 \mathrm{~kg}$.

Group II. The affected (7) calves were anesthetized, restrained, and prepared for aseptic surgery in the same manner as group (I). Animals were injected intravenously with 250 mg ethamsylate (Haemostop ${ }^{\circledR}$, Amoun Pharmaceutical Co., El-Obour City, Cairo, Egypt) 20 minutes prior to surgery for control of bleeding.

An elliptical skin incision connecting the two poles of the swelling was created. The incision was extended from the anal pole to the scrotal one, after which blunt dissection was advanced to separate the swelling from the surrounding skin and thin muscular layer without opening it. The ventral pole was separated first and the stump of the penis connected to the swelling was grasped with two haemostatic forceps and ligation was applied in between. Dissection was completed and advanced towards the anal pole. The dead space below the swelling was sutured by catgut and skin was closed by silk number 1-2 in interrupted manner started at the scrotal pole towards the anal one. The upper $5 \mathrm{~cm}$ of the skin wound was left opened and the swelling was excised to make a cone that was sutured to the skin in order to create wide urethral opening similar to urethrostomy (Fig.610). Aftercare was made in the same manner as group (I).

Each animal was monitored for 6 months and data were collected including duration of surgery, severity of bleeding, cost of surgery, cost of aftercare, complications, recovery period, body gain, durability, and penalizing of animal for low price as a result of surgery.

Examination of urine of affected animals was performed according to Benjamin (1984) and Kelley (1984).

\section{Results}

During surgery, the inner mucosal surface of the dilated urethra showed necrosis with presence of large amount of pus mixed with ammoniacal odor fluid. Moreover a pseudodiphtheritic necrotic tissue adhered to the mucosa was observed (Fig.4). Surgical procedure was easier in urethrostomy group than the group subjected to partial penile resection including the dilated urethra, as the bleeding was 
more controllable and violence of dissection could be avoided.

Regarding duration of surgery, it was observed that the duration of urethrostomy is shorter than that of partial penile resection, as urethrostomy lasted 20-55 minutes with a mean of 36.25 minutes controversial to partial penile resection that lasted 60-110 minutes with a mean of 85.71 minutes (Table 1). Moreover the bleeding was so extensive in partial penile resection technique when compared to the urethrostomy one.

With respect to the cost of surgery and aftercare, it was found that the cost of urethrostomy is $75 \%$ that of partial penile resection, but the cost of its aftercare was twice that of partial penile resection (Table 1).

Regarding complications, urine scald was observed in both groups but the incidence of other complications like cystitis and abscess formation was higher in urethrostomy group (3 calves) when compared to partial penile resection group (one calf) as shown in Table 1.
Results showed that recovery period was nearly the same in urethrostomy and partial penile resection groups (14.37 and 14.28 days respectively) (Table 1).

Monitoring of the weights of operated animals 6 months post surgery revealed that the mean body gain was $82 \mathrm{~kg}$ in partial penile resection group and $77.14 \mathrm{~kg}$ in urethrostomy group. However three animals were sold and evaluation of their body gains and weights was not available 6 months after surgery.

The created urethral opening was satisfactory in partial penile resection group than urethrostomy group as two animals of group (I) required widening of the urethrostomy fistula 4 months post surgery as a result of stricture of the fistula orifice (Table 1).

With respect to penalizing the animal at markets for low price at sale, both groups penalized and considered as low quality animals as a result of presence of both urine scald and urethral fistula as these animals became unfit for breeding (Table 1).

Table (1): Number of operated animals and duration of surgery, severity of hemorrhage, cost of surgery and aftercare, complications, weight before and 6 months after surgery, durability, and sale blemishing.

\begin{tabular}{|c|c|c|c|c|c|c|c|c|c|c|c|c|}
\hline & 䎡离 & 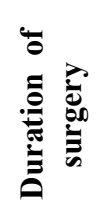 & 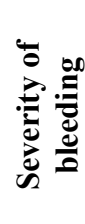 & 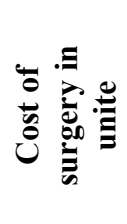 & 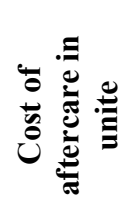 & ن & 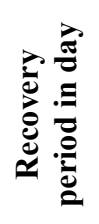 & 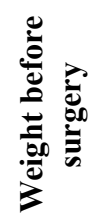 & 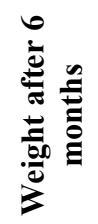 & 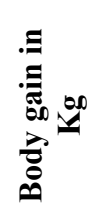 & 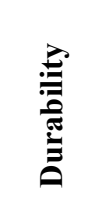 & 资 \\
\hline \multirow{8}{*}{ 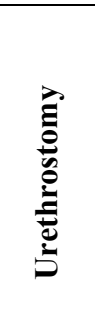 } & 1 & 20 & - & 150 & 100 & $\mathrm{C}$ & 15 & 60 & 115 & 55 & $>6 \mathrm{~m}$ & + \\
\hline & 2 & 25 & - & 150 & 0 & - & 10 & 60 & 145 & 85 & $>6 \mathrm{~m}$ & + \\
\hline & 3 & 35 & - & 150 & 0 & - & 10 & 80 & & & $*$ & \\
\hline & 4 & 50 & - & 150 & 0 & - & 10 & 120 & 210 & 90 & $>6 \mathrm{~m}$ & + \\
\hline & 5 & 45 & - & 150 & 100 & $\mathrm{C}$ & 25 & 100 & 180 & 80 & $4 \mathrm{~m}$ & + \\
\hline & 6 & 35 & - & 150 & 40 & A & 25 & 80 & 160 & 80 & $4 \mathrm{~m}$ & + \\
\hline & 7 & 55 & - & 150 & 0 & - & 10 & 120 & 200 & 80 & $>6 \mathrm{~m}$ & + \\
\hline & 8 & 25 & - & 150 & 0 & - & 10 & 60 & 130 & 70 & $>6 \mathrm{~m}$ & + \\
\hline \multirow{9}{*}{ 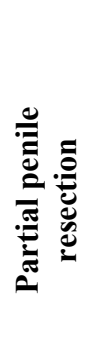 } & Mean & 36.25 & - & 150 & 30 & 3 & 14.37 & 97.14 & 162.8 & 77.14 & & \\
\hline & A & 70 & + & 200 & 0 & - & 15 & 60 & 135 & 75 & $>6 \mathrm{~m}$ & + \\
\hline & B & 80 & + & 200 & 0 & - & 15 & 60 & 140 & 80 & $>6 \mathrm{~m}$ & + \\
\hline & $\mathrm{C}$ & 90 & + & 200 & 0 & - & 15 & 60 & 145 & 85 & $>6 \mathrm{~m}$ & + \\
\hline & D & 110 & + & 200 & 100 & $\mathrm{C}$ & 20 & 120 & 0 & & * & \\
\hline & E & 100 & + & 200 & 0 & - & 15 & 120 & 220 & 100 & $>6 \mathrm{~m}$ & + \\
\hline & $\mathrm{F}$ & 90 & + & 200 & 0 & - & 10 & 120 & 0 & & $*$ & \\
\hline & G & 60 & + & 200 & 0 & - & 10 & 80 & 150 & 70 & $>6 \mathrm{~m}$ & + \\
\hline & Mean & 85.71 & + & 200 & 14.28 & 1 & 14.28 & 88.57 & 158 & 82 & & \\
\hline
\end{tabular}

$A=$ Abscess,$C=$ Cystitis, $\mathbf{m}=$ Month, and * means that the animal did not complete the study. 

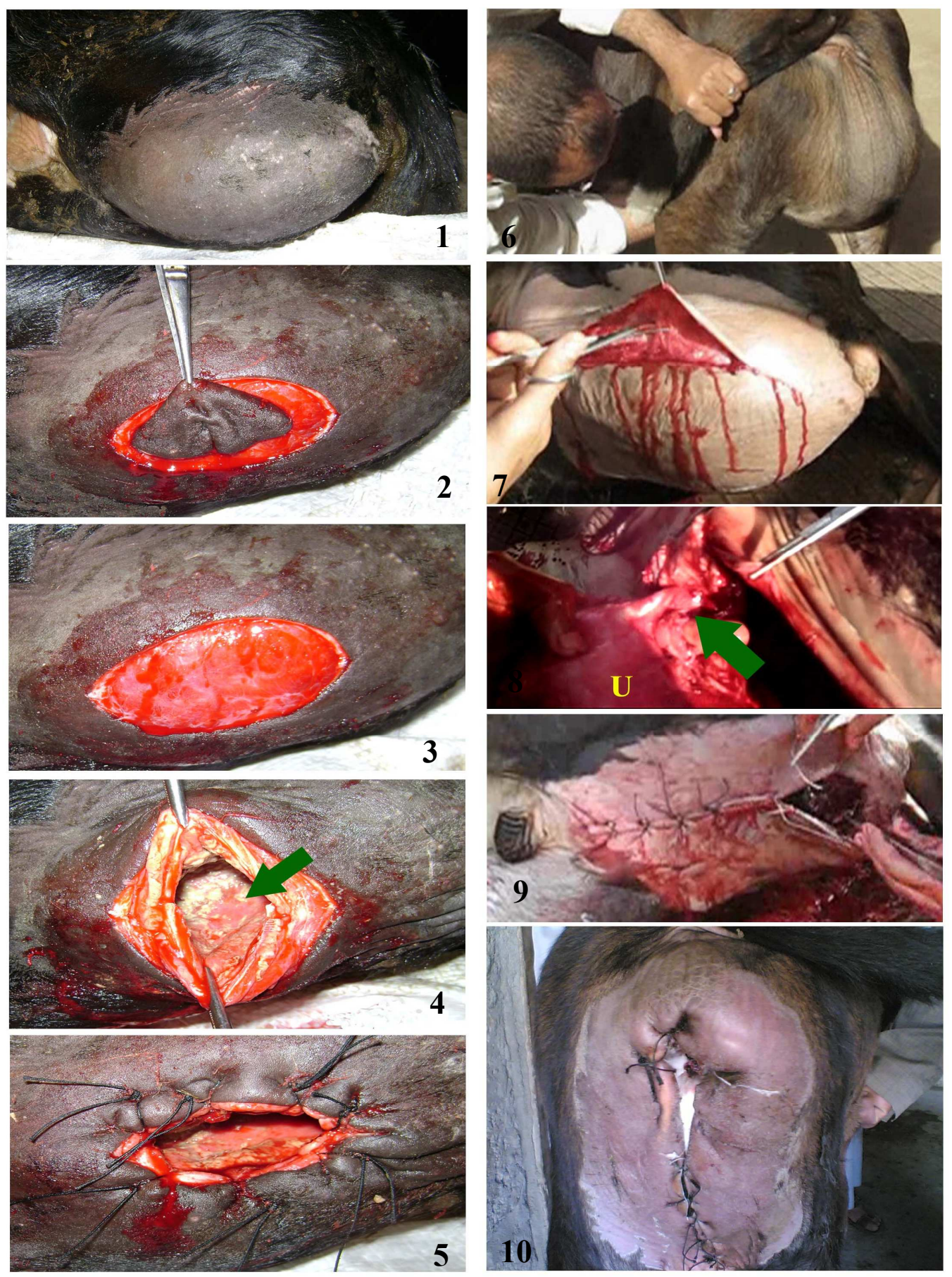

Figure (1): A calf with congenital urethral dilatation (group I, 3) prior to urethrostomy

Figure (2): The same calf after elliptical incision of the skin

Figure (3): The same calf after elliptical excision of the skin

Figure (4): The same calf after elliptical excision of the thin superficial muscular layer and urethra, arrow denotes necrosis and infection of urethral wall

Figure (5): Final shape of animal after urethrostomy

Figure (6): A calf with congenital urethral dilatation (group II, D) prior to surgical excision

Figure (7): Elliptical incision of skin and blunt dissection of the thin superficial muscle over the urethra

Figure (8): Complete dissection of the swelling and exposure of the penis ventral to it for ligation, arrow denotes the penis and $U$ means dilated urethra

Figure (9): the animal after suturing of the skin and fixation of the cone of dilated urethra ventral to anus Figure (10): the same animal after 7 days. 


\section{Discussion}

One of the most important aims of veterinary surgery in our locality is to save animal protein for our population, and at the same time it should be on economic base. From this point of view, certain Egyptian literatures discussed feasibility of treatment of certain surgical affections in our localities and the outcome of treatment of such affections (Abdel Fattah and Saleh, 2005). However the current study was conducted on a rare affection that infrequently reported in literatures and in general, treatment of such cases is directed towards correction of such affection when it is possible (Karras et al., 1992; Gasthuys et al., 1996; GeCcelep and Alkan, 2000).

Comparison between the two techniques revealed longer duration of surgery in group (II) than group (I) that can be explained in the light of severe adhesion between the dilatation and surrounding tissue as a result of prolonged duration of affection in addition to severe bleeding that required longer time for control, despite pre-surgical injection of hemostatic drug. Regarding the cost of surgery it was lower in urethrostomy technique than partial penile resection one, but the economical net revenue of surgical interference in both groups was high (Abdel Fattah and Saleh, 2005). Despite of the higher cost of aftercare in group (I) than group (II), the actual cost of aftercare was zero in both groups, but the represented cost involved treatment of complications that was the main reason of high cost of aftercare in both groups.

The observed complications in both groups agree with that mentioned by Anderson et al., (1993); Van Metre et al., (1996); Stone et al., (1997); Parsons et al., (1998); Susan (2003). However the incidence of cystitis was higher in urethrostomy group than partial penile resection one and it might be related to pooling of urine caudoventral to the ventral commeasure of urethrostomy opening. However, the observed cases of cystitis required prolonged duration of treatment with massive dose of antibiotic, diuretic, and anti-inflammatory that increased the overall cost of aftercare and recovery period and at the same time affected animals had lower body gain even after 6 months. Aside from complications, both groups had the same aftercare cost and duration of recovery was 5 days shorter in urethrostomy group. Stenosis of urethrostomy opening is a common complication of urethrostomy that may require reopening many times for establishment of a stoma despite it may be patent for 1-8 years in some studies
(Susan, 2003) but generally it has limited success rate (Haven et al., 1993; Van Metre et al., 1996). Stenosis was recorded in two calves in group (I) and it might be due to many factors as invasive approach into the urethra that is associated, unfortunately, with an increased risk of stricture formation, urethral scarring, and predisposition for re-obstruction (Harari, 2003); abscess formation in one calf and its invasive treatment; pooling of urine caudoventral to the ventral commeasure of urethrostomy with continuous irritation of the skin around the stoma and formation of over granulating tissue; and finally the direct contact of the two lips of the wound via pressure of the medial aspect of the two thighs. On the contrary, partial penile resection technique did not associated with stenosis despite surgical procedure in this group was more invasive and this can be explained in the light of the higher position of the created stoma (directly under anus) that prevented direct contact of wound lips, accumulation of urine at ventral commeasure, and subsequent abscess formation. Accordingly the stenosis might be related to the position of stoma rather than being related to the invasiveness of the surgical procedure. However, the observed high success rates with both techniques agrees with that given by Susan (2003) and disagree with that of Haven, et al., (1993); Gasthuys et al., (1996); Van Metre et al., (1996).

Clinical signs of cystitis included painful frequent urination with voiding of small amount of urine each time. Rectal examination revealed that the bladder wall was thick and painful to some extent (Radostits et al., 2000). Urine of affected animals was turbid and had strong ammoniacal odor. Moreover, microscopic examination of urine sediment revealed presence of erythrocytes and leucocytes (Benjamin, 1984; Kelley, 1984; Radostits et al., 2000).

Animals of both groups were penalized for low price at markets as a result of urine scald, loss of breeding capability and this agrees with Van Metre, et al., (1996) and Susan (2003), however the results were satisfactory to owners as the appetite of animals improved after surgery, fluctuation of temperature disappeared and body gain was accepted although it was lower than normal animals of the same age. Moreover, it is expected that fattening of these animals to slaughtering weights adopted in our markets would increase the economical net revenue of these animals and this agrees with that mentioned by Abdel Fattah and Saleh 
(2005). Although both techniques were satisfactory, and despite partial penile resection technique was associated with higher surgical cost, more severe bleeding, and invasive surgical procedure, it can be considered superior to urethrostomy for treatment of congenital urethral dilatation as a result of lower incidence of complications, lower aftercare cost, and better body gain.

\section{References}

Abdel Fattah, M. and Saleh, S. (2005): The feasibility study of the most important surgical interferences in cattle and buffaloes. Egyptian Soc. Anim. Reprod. Fert., Seventeenth Annual Congr, Al Menia, 29 January - 2 February, 2005, 307-318.

Anderson, D. E.; Wallace, C. E.; Williamson, L. and Mahaffey, M. B. (1993): Urethral recess dilatation in a Charolais-cross bull calf. Can. Vet. J., 34: 234-235.

Benjamin, M. M. (1984): Outline of veterinary clinical pathology. The Iowa state Univ. Press., Ams., Iowa 50010, USA.

Gasthuys, F.; Martens, A. and De Moor, A. (1996): Surgical treatment of urethral dilatation in seven male cattle. Vet. Rec., 138(1):17-19.

Gasthuys, F.; Steenhaut, M.; De Moor, A. and Sercu, K. (1993): Surgical treatment of urethral obstruction due to urolithiasis in male cattle: a review of 85 cases. Vet. Rec., 133: 522-526.

GeCcelep, M. and Alkan, I. (2000): Congenital urethral dilatation in a male montaphon calf Case report. Isr. J. Vet. Med., 55 (1). Hall, L. W. and Clarke, K. W. (1981): Veterinary anesthesia. $8^{\text {th }}$ ed., ELBS, Baillere TindallLondon.

Harari, O. (2003): Tube cystotomy for treatment of urethral calculi and obstruction in pet pigs. Israel Vet. J., 58 (4).

Haven, M. L.; Bowman, K. F.; Engelbert, T. A. and Blikslager , A. T. (1993) : Surgical management of urolithiasis in small ruminants. Cornell Vet., 83: 47-55.

Javdani, G. M.; Dehghani, N. S. and Raayat, J. A. R. (2009): Congenital penile urethral aplasia in a 4-day-old calf. Iranian J. Vet. Res. Shiraz Univ., 10 (1):87-89.

Johnson, E. H.; Nyack, B. and Johnson, R. (1980): Urethral dilatation in a steer: a case report. Vet Med Small Anim. Clin. 75 (9):1429-1431.

Karras, S.; Modransky, P. and Welker, B. (1992): Surgical correction of urethral dilatation in an intersex goat. J. Am. Vet. Med. Assoc., 201(10):1584-1586.

Kelley, W. R. (1984): Veterinary Clinical Diagnosis. Bailliere Tindall Williams Clowes Limited, Beccles and London.

King, W. W.; Young, M. E. and Fox, M. E. (2002): Multiple congenital anomalies in a polled goat. Contemp. Top. Lab. Anim. Sci., 41 (5): 39-42.

Misk, N. A. (2008): Atlas of veterinary surgery. Assiut Univ. Press.

Parsons, D. A.; Lawhorn, B.; Walker, M. A.; Edwards, J. F. and Hague, B. A. (1998): Incomplete urethral obstruction associated with dilatation of the urethra, cystitis, and pyelonephritis in a Vietnamese pot-bellied pig. J. Am. Vet. Med. Assoc., 212(2): 262-264.

Radostits, O.; Blood, D. C.; Gay, C. and Hinchcleff, B. (2000): Veterinary medicine, A textbook of the diseases of cattle, sheep, pigs, goats, and horses. $9^{\text {th }}$ Ed., Saunders Company Ltd London.

Stone, W. C.; Bjorling, D. E.; Trostle, S. S.; Hanson, P. D. and Markel, M. D. (1997): Prepubic urethrostomy for relief of urethral obstruction in a sheep and a goat. J. Am. Vet. Med. Assoc., 210 (7): 939-941.

Susan E. Aiello (2003): Noninfectious Diseases Of The Urinary System In Large Animals, Urolithiasis in Ruminants, the Merk veterinary manual; $8^{\text {th }}$ Edition.

Tharp, V. L. and Venzke, W. G. (1954): Dilatation of the urethra in bull. J. Am. Vet. Med. Assoc., 269.

Van Metre, D.; Fecteau, G.; House, J. K. and George, L. W. (1996): Obstructive urolithiasis in ruminants: surgical management and prevention. Compend. Contin. Educ. Pract. Vet., 19: 275-289.

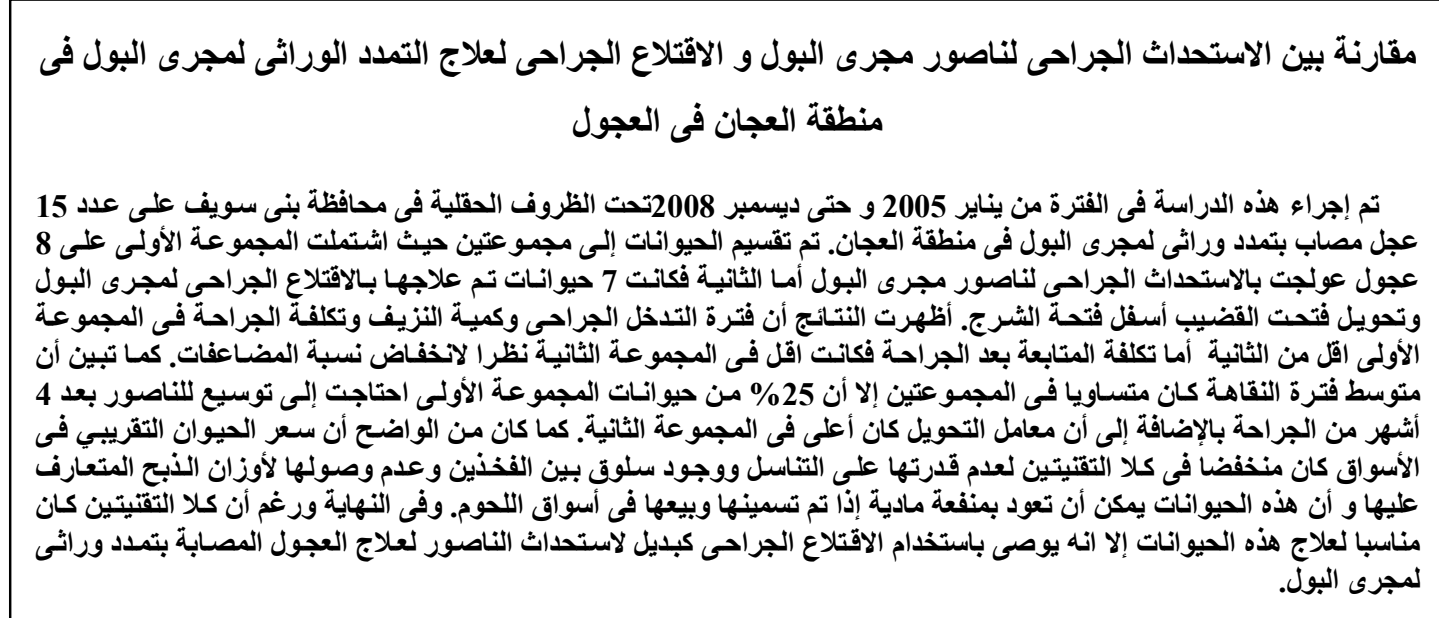

